\title{
La hostilidad y su relación con los trastornos cardiovasculares
}

\author{
Jorge Iacovella ${ }^{1}$ \\ Marisa Troglia ${ }^{2}$
}

\begin{abstract}
Resumen
Uno de los campos más desarrollados en el ámbito de la Psicología de la Salud es el de los aspectos psicológicos que intervienen en la enfermedad cardíaca. Dentro de los constructos psicológicos más estudiados se encuentra el de la hostilidad. Inicialmente componente del patrón de conducta tipo A, se han ido desarrollando diferentes formas de referirla, y por consiguiente, de medirla. En este artículo se analizan algunas de las nociones más citadas sobre la hostilidad y otras manifestaciones asociadas, los modelos explicativos de su relación con los trastornos cardiovasculares - en especial el de la hiperreactividad cardiovascular - y los instrumentos que se desarrollaron para su evaluación. Por último, se discuten las actuales implicancias del uso del constructo, así como las áreas de investigación a desarrollar para delimitar mejor este factor que ha sido referido en forma diversa, y consiguientemente ha reportado en los diferentes estudios resultados no siempre consistentes entre sí.

Palabras-Clave: Enfermedad cardíaca; Patrón de conducta tipo A; Hostilidad; Ira; Modelo de hiperreactividad cardiovascular.
\end{abstract}

\section{Hostility and its relation with cardiovascular disorders}

\begin{abstract}
One of the most highly developed fields in the Health Psychology area is that of the aspects involved in causing cardiac disease. Within the more thoroughly studied psychological constructs is the hostility one, component of the type A behavior pattern. Different ways of referring to this construct and of measuring it have been developed. This article analyzes some of the most pervasive notions about hostility and other related manifestations, the models explaining its relationship with cardiac disorders, especially the cardiovascular hypereactivity disorder, and the instruments designed for its assessment. Finally, current implications and fields of potential research are discussed, in order to further delineate this factor which has been referred to in a diverse fashion, thus showing different and not always consistent results in previous studies.
\end{abstract}

Keywords: Cardiac disease; Type A behavior pattern; Hostility; Anger; Cardiovascular hypereactivity model.

\section{Introducción}

La relación entre estados, sucesos o procesos psicológicos y la salud orgánica ha sido ampliamente estudiada y referida en la literatura occidental. En un recorrido que va desde la concepción aristotélica del cuerpo como forma del alma, pasando por una variedad de explicaciones tentativas de la relación mente-cuerpo, hasta llegar a los modelos actuales que delimitan con mayor precisión la relación entre unos constructos psicológicos específicos con enfermedades orgánicas determinadas, casi todos estos modelos tienen en común enfatizar el papel de las emociones en la salud, sea por su valor adaptativo, sea participando en la génesis de las enfermedades, contribuyendo a su permanencia o favoreciendo su agravamiento.

En el caso de la salud coronaria, los factores tradicionales de riesgo no llegan a explicar ni la mitad los casos de trastornos cardiovasculares (FernándezAbascal, Palmero \& Martín Díaz, 1998), a partir de lo

Endereço para correspondência:

${ }^{1}$ E-mail: jiacovel@mdp.edu.ar

${ }^{2}$ E-mail: mtroglia@mdp.edu.ar cual la relevancia del estudio de los llamados factores no tradicionales se ha incrementado notoriamente. Los vacíos que han dejado los factores clásicos hacen referencia al estrés psicosocial y a las diferencias individuales en la percepción de los estímulos ambientales estresantes. Estos factores psicológicos tienen un papel modulador sobre los factores clásicos de riesgo, los cuales son en su mayoría biológicos. Como ejemplo podemos citar el Proyecto Stanford de Intervención en el Riesgo Coronario (Gordon, 1998). Implementado en Estados Unidos de Norteamérica, este proyecto resulta de interés porque empleó una estrategia que incluye modificación radical de varios factores de riesgo mediante cambios en el estilo de vida combinados con terapia farmacológica, sin embargo, no incluyó el tratamiento de aspectos psicológicos como los que trataremos en este trabajo a pesar de que su relación con las enfermedades cardiovasculares ha recibido hasta el momento un amplio apoyo empírico.

En el contexto mencionado, la ira es de las 
emociones más estudiadas, por su participación en la activación fisiológica y en particular por ser uno de los componentes del constructo en estudio. Por encontrarse fuertemente asociado con la enfermedad cardiovascular, este constructo ha recibido el nombre de patrón de conducta tipo A (PCTA) (Friedman \& Rosenman, 1974). En la actualidad, y devenido de este, es más frecuentemente estudiado el complejo ira-hostilidad.

\section{Características de la ira y la hostilidad}

A raiz de las críticas hechas al patrón de conducta tipo A (PCTA) como predictor de trastornos cardiovasculares se comenzó a investigar un nuevo patrón de predisposición conductual que indicara más eficazmente la posibilidad de presentar un trastorno cardiovascular.Ya que la hostilidad se mostrara como el componente más tóxico y nocivo del PCTA en tanto indicador de riesgo coronario, la atención se centró sobre la dimensión hostil. Surgió así el complejo irahostilidad, amplio constructo que engloba distintos aspectos (Barefoot, 1992), un componente cognitivo (hostilidad), un componente afectivo-emocional (ira) y un componente conductual (agresión).

También en busca de nuevos constructos con mayor capacidad predictiva Spielberger, Krasner y Solomon (1988) formularon el sindrome ira-hostilidadagresión (AHÍ) como complejo asociado al riesgo de enfermedad coronaria (EC). El mismo incluye la ira en tanto estado y en tanto rasgo, así como también la expresión (ira-in e ira-out) y el control de la ira.

El posible mecanismo fisiológico implicado en la relación entre el complejo ira-hostilidad y los trastornos cardiovasculares podría ser la hiperactivación de los ejes simpático-adrenomedular y adenohipofisiarioadrenocortical. Parece que tanto la alta activación puntual y repetida como la hiperactivación mantenida de estos ejes son las responsables de que los sujetos hostiles tengan una mayor incidencia de enfermedades coronarias.

La hostilidad es considerada por los investigadores como una variable compleja y de difícil conceptualización, de la que carecemos de una definición comprensiva (Rosenman \& Palmero, 1998). De la misma forma que ocurrió con el PCTA se plantea la posibilidad de que el constructo hostilidad tenga un carácter multidimensional, siendo sólo alguno de sus componentes el elemento que posea una naturaleza dañina en el desarrollo de la enfermedad cardiovascular (Palmero \& Codina, 1996).

La Hostilidad es el resultado de las influencias que puede haber recibido un sujeto por parte de su medio ambiente a lo largo de su vida, ya que el factor genético si actúa, lo hace de modo muy reducido. Los sujetos que presentan hostilidad se caracterizan por esperar lo peor de los demás, estar siempre a la defensiva, crear ambientes de tensión y competitividad y percibir al entorno como un espacio de lucha para lograr objetivos.

\section{El síndrome ira - hostilidad - agresión (AHÍ)}

Para Smith (1992) las nociones de ira, agresión y hostilidad están íntimamente relacionadas. Es esta mutua relación lo que impide una fácil diferenciación. Sin embargo, la distinción entre afecto, cognición y conducta, permite un marco de referencia útil para establecer diferencias entre las nociones.

Las definiciones de hostilidad consideran que ésta implica la presencia de una serie de creencias, expectativas y actitudes negativas relativamente duraderas y estables acerca de las personas y de las cosas. Predominan como creencias el cinismo y las atribuciones hostiles, se piensa que las demás personas son generalmente mezquinas, egoístas, deshonestas, antisociales e inmorales. Las demás personas constituyen una fuente de amenaza y tienen intenciones malevolentes con respecto a nosotros (Barefoot, 1992). Otras creencias consisten en considerar que todo lo que uno hace, piensa y dice es correcto, que los demás son ignorantes e ineptos (Friedman, 1983) y que los recursos disponibles son escasos y finitos, por lo que hay que "luchar" con los demás para poder cubrir las necesidades (Price, 1982). Asociadas aparecen expectativas negativas como desconfianza o sospecha. No se confía en la bondad de los otros, estimando que es poco probable que éstos cumplan con sus obligaciones y sean agradables y cooperativos sin un propósito determinado (Barefoot, 1992). También puede manifestarse una actitud negativa caracterizada por enemistad, negativismo, resentimiento (Smith, 1994).

A su vez, la ira refiere a una emoción displacentera compuesta por sentimientos de variable intensidad, conlleva una experiencia subjetiva (sentimientos y pensamientos), una activación fisiológica y neuroquímica y un determinado modo de expresión o afrontamiento. Las situaciones que la desencadenan están relacionadas con condiciones en las que somos heridos, engañados o traicionados, tienen que ver con el ejercicio de un control físico o psicológico en contra de nuestra voluntad o se nos impide alcanzar una meta a la que consideramos que tenemos derecho. También pueden actuar como desencadenantes de la ira la estimulación aversiva (dolor físico, exposición al humo de los cigarrillos, olores fuertes y altas temperaturas) o la falta de un mínimo de estimulación, tales como restricción física o psicológica (Lazarus, 1991).

El componente subjetivo o experiencial de la ira suele ser llamado experiencia de ira. Puede variar en intensidad, oscilando desde una irritación leve hasta la 
furia o rabia. Parece que la experiencia de ira es más intensa cuando el individuo tiene baja tolerancia a la frustración, cuando la pérdida de aquello que valoramos ocurre inesperadamente, cuando es percibida como excesivamente injusta y cuando compromete cualquier aspecto muy valorado por el individuo.

Spielberger y colaboradores (1985) consideran que la experiencia de ira puede estudiarse a partir de dos manifestaciones, ira estado e ira rasgo. La primera, es la experiencia temporal de sentimientos subjetivos de tensión, enfado, irritación o furia en respuesta a una serie de eventos; suele ir acompañada de aumentos en la activación fisiológica. La ira rasgo se refiere a la disposición a experimentar episodios de ira de forma frecuente y/o pronunciada, bien por la percepción de un amplio rango de situaciones como provocadoras, bien por la predisposición a experimentar elevaciones más intensas, bien debido a la interacción de ambos factores. La ira estado y la ira rasgo están íntimamente relacionadas. La activación fisiológica y neuroquímica se caracteriza por incrementos en la activación del sistema nervioso autónomo (SNA), del sistema endocrino y de la tensión muscular, así como por una serie de cambios respiratorios.

El modo de afrontamiento o de expresión de ira se conoce con los términos de estilos de afrontamiento de la ira o expresión de ira. Spielberger y colaboradores (1985) consideran que hay dos modos fundamentales de expresión de la ira, interna y externa. La ira interna se refiere a la tendencia a suprimir los sentimientos de enfado, y a veces también los pensamientos, que se experimentan, no expresándolos abiertamente. Supone realizar un esfuerzo por parte del sujeto para que el enfado no sea observable; por tanto, como la energía generada por la ira no se expresa, puede repercutir internamente. La ira externa hace referencia a la tendencia a manifestar abiertamente los sentimientos de ira que se experimentan. Hay dos formas de expresión de la ira externa, una forma comunicativa o asertiva, que incluye expresiones no amenazantes de los sentimientos de ira o formas socialmente adecuadas de expresarla, y una forma agresiva, que incorpora la expresión de ira con el propósito de infligir daño. Johnson (1990) propone tres estilos diferentes de afrontamiento para disminuir la sensación displacentera de la emoción de ira que son la supresión de la ira (anger-in), la expresión de la ira (angerout) y, por último, el control de la ira (anger-control).

En el estilo de supresión de la ira el sujeto afronta la situación reprimiendo la expresión verbal o física. En la expresión de la ira el sujeto manifiesta conductas airadas, verbales o físicas, hacia sujetos $\mathrm{y} / \mathrm{u}$ objetos, pero sin la intención de producir daño a una persona. Finalmente, en el control de la ira el sujeto tratará de canalizar su energía emocional y proyectarla hacia fines más constructivos, para llegar a una

Psico-USF, v. 8, n. 1, p. 53-61, Jan./Jun. 2003 resolución positiva del conflicto.

Este último estilo - el control de la ira - sería la alternativa más adecuada para reducir la probabilidad de riesgo de coronariopatías. En cambio, los sujetos que presentan el estilo supresión de la ira son muy susceptibles de sufrir trastornos cardiovasculares. En cuanto a los sujetos que presentan un estilo de expresión de la ira, además de suponer una activación fisiológica mayor, es dado creer que de este modo socavan el apoyo social, lo cual redundaría en una mayor vulnerabilidad a las enfermedades en general.

Por fin, el término agresión suele utilizarse para aludir a la conducta o conductas destructivas o punitivas dirigidas hacia otras personas u objetos. Estas conductas suelen ir acompañadas por una serie de cambios en la expresión facial, en el tono y en el volumen de voz, en la postura y en el movimiento, que pueden ser observados directamente. Aunque el término agresión suele emplearse para designar a conductas de ataque que son destructivas o dañinas, también pueden ser consideradas conductas de agresión las conductas de omisión voluntaria y consciente que hacen que otra persona pueda recibir un estímulo aversivo.

Pero, tanto la ira como la agresión, esto es, las dimensiones subjetiva y conductual, son variables inestables y transitorias en el tiempo. Por consiguiente, los estudios referidos a la relación entre el complejo IraHostilidad, o el Síndrome AHI y las coronariopatías, se han centrado en la consideración de la dimensión cognitiva, es decir, la hostilidad. Esta constituye el aspecto cognitivo, es estable y duradera en el tiempo, razón por la que se erige como el factor a evaluar para comprender la relación entre la enfermedad cardiovascular y los proceso emocionales.

Diferentes estudios han señalado que los sujetos hostiles son más proclives que los no hostiles a desarrollar coronariopatías e, incluso, que situaciones de ira pueden precipitar los procesos de EC (Matthews, Glass, Rossenman \& Bortner, 1977). Se ha puesto de manifiesto que los procesos cardiovasculares patológicos estarían asociados con el potencial para la hostilidad (Dembroski, Mac Dougall, Costa \& Grandits, 1989), y que el pobre manejo de los sentimientos hostiles, especialmente cuando son suprimidos, sería más destructivo (Spielberger, 1990).

\section{El proceso emocional de la ira-hostilidad}

Se analizará este proceso entendiendo que no son las situaciones en sí las que causan una determinada reacción emocional, sino que de acuerdo con la evaluación situacional que haga el individuo se manifestarán diferentes formas y diferentes grados de emoción. Así, en el modelo de Lazarus (Lazarus \& 
Folkman, 1984), la aparición del estrés y otras reacciones emocionales están mediatizadas por un proceso de valoración cognitiva. En una primera instancia la valoración es sobre las consecuencias que la situación tiene para ella misma - valoración primaria y luego, si el resultado de la valoración es de amenaza o desafío, la persona evalúa los recursos de que dispone para evitar o reducir las posibles consecuencias negativas de esta situación - valoración secundaria.

Fernández-Abascal y Palmero (1999) proponen entender a la ira-hostilidad como un unidad en la que cada una de estas manifestaciones son componentes del mismo proceso emocional. El proceso, como en todas las emociones, se inicia con la evaluación (cognición) de un estímulo (percepción) que realiza un sujeto con un determinado estado afectivo (afecto), si el estímulo es procesado como un candidato a desencadenar la emoción de ira, entonces la persona experimentará dicha emoción. Así se activa fisiológicamente, se produce una cierta disposición actitudinal hostil (cognición) y se activan tendencias a la acción que eventualmente darán lugar a una forma de afrontamiento, la agresión. La respuesta fisiológica se caracteriza por un aumento de la activación simpática, la hostilidad modula la respuesta fisiológica y las tendencias a la acción (aumentándolas). En cuanto a la acción, el sujeto la llevará a cabo si estima que sus recursos para la agresión son suficientes y lo considera pertinente. Si no se dá alguna de estas condiciones entonces reprime el impulso y consecuentemente se incrementará la hostilidad (también podría ser incrementado el sentimiento de ira), de este modo se produce también un aumento de la activación fisiológica o su prolongación en el tiempo.

\section{Modelos explicativos de la relación AHÍ-ECV}

Existen diversos modelos que explican los nexos entre el síndrome AHÍ y la salud en general, quedando implicada en ellos la relación del mencionado síndrome con las enfermedades cardiovasculares. El modelo de vulnerabilidad somatopsíquica de Krantz y Durel (1983) para el que las diferencias biológicas entre los individuos serían las responsables de las diferencias psicológicas, entre ellas de la manifestación de agresión, hostilidad e ira. Así habría personas biológicamente predispuestas por una mayor responsividad cardiovascular y también un patrón psicológico en el que está presente el síndrome AHÍ.

Un segundo modelo, el modelo de la vulnerabilidad psicosocial (Smith \& Frohm, 1985), propone que la hostilidad de algunas personas les genera conflictos interpersonales $y$, en consecuencia, un bajo nivel de apoyo social, lo que constituirá un factor de riesgo para sufrir trastornos cardiovasculares. Asimismo, el modelo conducta-salud, de Leiker y Hailey (1988) sugiere que las personas hostiles presentan además malos hábitos de salud. Se han encontrado correlaciones entre hostilidad y falta de ejercicio físico, poco cuidado personal, episodios de consumo excesivo de bebidas alcohólicas, tabaquismo y otras conductas de riesgo cardiovascular. Además, las mismas características psicológicas del síndrome AHÍ se hallan relacionadas con una baja adherencia a los tratamientos médicos.

El modelo de reactividad psicofisiológica de Williams, Barefoot y Shekelle (1985) considera a la hostilidad como una causa contribuyente al desarrollo de la enfermedad cardiovascular. Habría un incremento en las respuestas fisiológicas, tanto en intensidad como en cantidad, al aumentar la frecuencia de los episodios de ira $y$ encontrarse el individuo en un estado de hipervigilancia ante el entorno social. En el apartado siguiente se presenta con más detalle este modelo.

Por último, el modelo transaccional de Smith y Pope (1990) integra y extiende el alcance de las aproximaciones basadas en la reactividad psicofisiológica y en los apectos psicosociales. En primer término, sostiene que las personas hostiles interpretan el accionar (y las intenciones) de los otros de tal manera que terminan por provocar conflictos interpersonales, perdiendo, entonces, apoyo social. En segundo término, la alta reactividad psicofisiológica surge de la hipersensibilidad de las personas hostiles ante las situaciones sociales potencialmente estresantes, y además ante los estresores sociales que ellos mismos han provocado.

Todos estos modelos encuentran buen apoyo evidencial, pero también son susceptibles de revisión, y consecuentemente de nuevas investigaciones, ya que ninguno de ellos cuenta con argumentos empíricos concluyentes para desplazar a los demás. A continuación consideraremos más extensamente la reactividad psicofisiológica por ser una de las más estudiadas y aceptadas explicaciones de la contribución psicológica a la enfermedad cardiovascular.

\section{Reactividad psicofisiológica}

Aquí se amplía la explicación de los mecanismos subyacentes a la correlación entre el constructo y las enfermedades cardiovasculares. Este modelo, que está basado en el análisis de la reactividad psicofisiológica, recibe el nombre de modelo de la hiperreactividad cardiovascular o reactividad cardiovascular, $\mathrm{y}$, tal como lo caracterizan Fernández-Abascal, Palmero y Martín Díaz (1998), propone la existencia de una especificidad individual de respuesta psicofisiológica, una estereotipia personal, que denota la propensión que presentan algunas personas a mostrar patrones fisiológicos de 
respuesta cardiovascular incrementados en relación a los que presenta el resto de la población. Estos patrones para ser considerados reactivos, tienen que ser estables y fiables, pues deben mantenerse a lo largo del tiempo y de las situaciones para una persona dada.

La hipótesis central del denominado modelo de reactividad psicofisiológica sostiene que esta hiperreactividad cardiovascular y neuroendocrina, principalmente catecolamínica, se encuentra mediada por una activación más pronunciada del Sistema Nervioso Simpático (SNA). Fernández-Abascal y Martín Díaz (1999), al referirse a este modelo, dicen que las personas hostiles experimentan más frecuentemente intensos episodios de ira y se encuentran en un estado permanente de hipervigilancia ante el entorno social. Estas conductas son las que producen la activación excesiva y frecuente del SNA. Es importante destacar que la reactividad se presenta tanto en las personas que ya han desarrollado una enfermedad, como en sujetos con una predisposición a su desarrollo; lo que la constituye en un importante factor de riesgo para llegar a enfermar.

El modo en que algunas personas responden al estrés con su sistema cardiovascular, está definido por una exagerada activación beta-adrenérgica $y$ un decremento sinergético. Entonces, estos sujetos, ante las situaciones estresantes, muestran índices más elevados en su presión arterial, frecuencia cardiaca o actividad vasomotora. En el mismo sentido Obrist (1981) halló difencias significativas para la reactividad vascular en las personas de acuerdo con las respuestas beta-adrenérgicas a tareas que exigían afrontamiento activo, así se comenzó a hablar de sujetos reactores (High reactors) y no reactores (Low reactors).

De acuerdo con Fernández-Abascal, Palmero y Martín Díaz (1998) las características definitorias de la reactividad cardiovascular, que la diferencian de otros constructos como la labilidad autonómica, son la magnitud de la respuesta de reactividad cardiovascular que se refiere a una doble característica, por una parte a la amplitud de la respuesta dada por una persona y, por otra, a la incidencia que estos patrones de respuesta tienen en la población; la estabilidad temporal de la reactividad cardiovascular que está referida a la constancia en el tiempo de los patrones de respuesta fisiológicos que presentan las personas reactivas. Esto es, si las personas que se manifiestan con reactividad cardiovascular en una evaluación, la mantienen en las subsiguientes. También, las características emocionales de las personas reactivas que son relativas a los constructos que ya hemos comentado en la introducción (PCTA e ira-hostilidad); los parámetros psicofisiológicos utilizados en la evaluación, o la forma en que se determina la reactividad cardiovascular es de suma importancia, para

Psico-USF, v. 8, n. 1, p. 53-61, Jan./Jun. 2003 su correcta evaluación y para que los resultados obtenidos sean comparables con los datos existentes. Y por último, las condiciones bajo las que se realizan las medidas, es decir, el tipo de condiciones, tareas o situaciones que se han utilizado para la medida de la reactividad cardiovascular. Principalmente asociadas a procedimientos de laboratorio, - aunque también se realizan en entornos naturales - de acuerdo al tipo de afrontamiento que exigen de los sujetos podemos clasificar las condiciones como activas (tareas de tiempo de reacción, de aritmética mental, etc.) o pasivas (cold pressor). Las tareas que exigen afrontamiento activo son más propicias para evaluar la reactividad cardiovascular ya que en ellas se manifiesta la diferencia entre sujetos reactivos y no reactivos porque estimulan la acción beta-adrenérgica sobre el corazón. Debido a la especificidad del síndrome AHÍ, los estudios de laboratorio han sugerido que la reactividad cardiovascular se limita a situaciones de estrés social, con resultados dudosos en otro tipo de situaciones estresoras (Fernández-Abascal \& Martín Díaz, 1999).

Con respecto a los efectos de la hiperreactividad en el sistema cardiovascular, existen numerosos estudios que evidencian la nociva asociación existente. En el meta-análisis que precede a su estudio sobre las relaciones entre hostilidad y colesterol, Calvo, Alemán y Ojeda (1998) sostienen que es plausible que una mayor activación del sistema nerviosos autónomo, en respuesta a estresores, juegue un papel relevante para posibles daños del endotelio como también del consiguiente depósito de lípidos en la pared arterial. Los efectos de las catecolaminas para mediar tanto en el aumento de la activación cardiovascular como en la movilización de los lípidos de los depósitos grasos, podrían ser críticos para incrementar la aterosclerosis coronaria entre individuos con comportamientos procoronarios. Otro mecanismo, que estaría implicado está vinculado con el eje pituitario-adrenal; el exceso crónico de corticoesteroides produce una cantidad de efectos que podrían tener importancia en la aterogénesis. Dichos efectos consisten en un incremento de los lípidos circulantes, de la aterosclerosis (observada en modelos animales) y de la proporción de células endoteliales dañadas o muertas. La conclusión es que la combinación de la hiperreactividad de catecolaminas y cortisol durante situaciones de estrés, propia de individuos con ciertas características comportamentales y psicológicas, presentes de forma crónica, podría jugar un papel clave en el desarrollo de la aterosclerosis. Siguiendo este analísis encontramos que la reactividad plaquetaria al estrés psicológico puede ser el principal mecanismo responsable de los accidentes coronarios (Markovitz, 1998).

Entre los estudios pioneros sobre la reactividad cardiovascular se pueden citar los de Obrist (1981), quien presenta una investigación sistemática de las influencias 
conductuales sobre la covariación de los procesos cardiovasculares y somatomotores. Específicamente, estos estudios indagan la relación entre la actividad muscular y la tasa cardíaca para diferentes manipulaciones conductuales (tareas de afrontamiento activo y pasivo de situaciones estresantes), así como examinan los mecanismos del sistema nervioso autónomo que están involucrados. Se ha observado que en muchas situaciones la interacción cardíaco-somática fue apareada de manera metabólicamente apropiada, con cambios en la respuesta cardíaca, siendo ante todo vagalmente mediada. Sin embargo, las situaciones que requirieron un afrontamiento activo para el control de estímulos nocivos llevaron a respuestas de tasa cardíaca que resultaron excesivas para el involucramiento somático. Spielberger, Krasner y Solomon (1988) han constatado una correlación negativa significativa entre la sub-escala Ira Hacia Dentro de la Escala de Expresión de la Ira y la disminución de la luz arterial. En esa misma línea, Boltwood, Taylor, Boutte Burke, Grogin y Giacomini (1992), en un estudio de doce pacientes con isquemia miocardial sintomática, hallaron que el recuerdo de un reciente suceso provocador de ira daba lugar a una vasoconstricción aguda de las arterias coronarias previamente estrechadas. La ira aparecía significativamente correlacionada con la disminución tanto de los diámetros mínimos como medios de las arterias coronarias, como se demostró mediante técnicas angiográficas cuantitativas.

Para Houston y Vavak (1991) la correlación positiva entre hostilidad y reactividad cardiovascular estaría determinada por el tipo de situación empleada para provocar la reactividad. La inconsistencia de los resultados hallados en la literatura aparecerían condicionados por el empleo de una serie de situaciones estresantes que son inocuas para provocar que las diferencias en la activación entre personas con altos y bajos niveles de hostilidad se pongan de manifiesto. Por esta razón, proponen el empleo de tareas que induzcan alto nivel de estrés psicosocial, en lugar de las que son habitualmente utilizadas (aritmética mental, efecto Stroop, cold pressor).

\section{Instrumentos de medición del constructo}

El tiempo que ha transcurrido desde la construcción de la mayoría de estos instrumentos resulta escaso aún para tener estudios longitudinales confiables, lo que explica porque la mayoría de ellos deben ser tomados con precaución en cuanto a su validez predictiva en el campo de la salud (FernándezAbascal \& Palmero, 1999). Entre los instrumentos más referidos para medir Ira pueden distinguirse aquellos surgidos de la clínica y con aplicación preferente en este campo y los surgidos para la investigación.
Entre los primeros se pueden mencionar, el Inventario Multidimensional de ira (Siegel, 1986) comprende cinco escalas, o sea, ira hacia adentro, ira hacia fuera, rango de situaciones elicitadoras de ira, punto de vista hostil e ira general. El Inventario de Ira Novaco (Novaco, 1975) proporciona un índice global del nivel de ira; existe una adaptación española (Martín \& Fernández-Abascal, 1994a). El Inventario de Control de la Ira (Hoshmand \& Austin, 1987) está compuesto de diez subescalas, a saber, ver abusos en otros, intrusión, degradación personal, traición de la confianza, malestar, control externo y coacción, abuso verbal, abuso físico, trato injusto y bloqueo de metas. Y finalmente, la Escala de Autoinforme de ira (Zelyn, Adler \& Myerson, 1972) que mide cinco subescalas, cuales sean, conciencia de ira, expresión de ira, culpabilidad, condenación de la ira y desconfianza.

En cuanto a los instrumentos surgidos principalmente para la investigación empírica podemos mencionar algunas. Las Escalas de Ira de Framingham (Haynes, 1978) miden cuatro aspectos de esta emoción, a saber, síntomas de ira, ira hacia adentro, ira hacia fuera y comunicación de la ira. La Escala de Ira hacia dentro y hacia fuera de Harburg y colaboradores (1973) incluye escalas para medir tres dimensiones, ira hacia adentro, ira hacia fuera y reflexión. El STAXI, Inventario de Expresión de la Ira Estado-Rasgo (Spielberberg, 1988) consta de ocho escalas, cuales sean, estado de ira, rasgo de ira, temperamento airado, reacción airada, control de la ira, ira hacia fuera, ira hacia dentro y expresión de ira. Si bien es de construcción bastante reciente, este instrumento ha concitado la atención de los investigadores y se ha desarrollado gran cantidad de investigación en torno a él.

Con respecto a la evaluación de la hostilidad los instrumentos más usados son seis. La Entrevista Estructurada de Rosenman (1974), incluye tres dimensiones, potencial de hostilidad, ira hacia adentro y hacia fuera. Este instrumento se construyó con el propósito de medir el PCTA y es el único que no se basa en autoinforme, está constituido por observación y preguntas. La Escala de Hostilidad de Cookle-Medley (1954) provee una información global de hostilidad, si bien pueden distinguirse diversos factores. El Cuestionario de Hostilidad y su Dirección (Foulds, Caine \& Creasy, 1960) posee cinco escalas, a saber, hostilidad hacia fuera, criticismo de otros, proyección de hostilidad engañosa, autocrítica y culpabilidad. La Escala de Hostilidad Manifiesta (Siegel, 1986), surgió en el ámbito de investigación y actualmente se usa en el campo clínico, mide la fuerza para expresar la hostilidad. El Cuestionario de Agresión desarrollado por Buss y Perry (1992), es una herramienta desarrollada para investigación empírica de los diversos 
componentes de la hostilidad: agresíon física, agresión verbal, ira (componente emocional) y hostilidad (componente cognitivo). Por último, el Inventario de Hostilidad de Buss-Durkee (1957) es utilizado en el campo de la investigación así como en el de la clínica y de la salud. Consta de siete subescalas, a saber, asalto o ataque, hostilidad indirecta, irritabilidad, negativismo, resentimiento, sospecha o recelo y hostilidad verbal. Hay una adaptación española del instrumento (Martín y Fernández-Abascal, 1994b).

\section{Conclusión}

En los últimos tiempos, se ha sugerido que el síndrome ira-agresión-hostilidad es el componente del PCTA que predispone a los sujetos a la enfermedad coronária. Sin embargo, hasta el momento sería apresurado establecer conclusiones definitivas respecto a la importancia que tiene dicha variable para explicar el padecimiento de enfermedades cardiovasculares. Las inconsistencias encontradas entre los resultados de distintos estudios hacen que algunos investigadores pongan en duda la solidez de la asociación existente entre el síndrome ira-agresión-hostilidad y la enfermedad coronária (García León, 1999).

Se ha planteado que estas inconsistencias podrían deberse al hecho de ignorar la naturaleza multidimensional del propio constructo ira-agresiónhostilidad. La existencia de numerosas definiciones y el desacuerdo o la falta de comunicación con respecto a la terminología parece haber producido cierta confusión al analizar los resultados de los distintos estudios. Además, en un primer momento se ha considerado que diferentes los instrumentos podían proporcionar la misma información con respecto al constructo; por el contrario, lo que se ha comprobado es que distintos instrumentos de medida parecen evaluar componentes o aspectos diferentes y que, de hecho, existen diferencias en el modo en que estos instrumentos se relacionan con la enfermedad coronária.

De la revisión de Smith (1992) se desprende que no podemos obtener conclusiones claras sobre la capacidad predictiva de esta variable sobre el desarrollo de enfermedades coronarias debido probablemente a que las diferentes medidas de hostilidad no son intercambiables. Sin embargo, en las dos importantes meta-análisiss realizadas sobre los estudios de las conductas, personalidad y emociones asociados con los trastornos coronarios, el de Booth-Kewley y Friedman (1987) y el de Matthews (1988) concluyen que la ira y la hostilidad son buenos predictores de la EC, siendo la hostilidad la más fuertemente asociada.

Como hemos visto, los procesos de referenciación de la ira y la hostilidad están en Psico-USF, v. 8, n. 1, p. 53-61, Jan./Jun. 2003 permanente dinámica y, en consecuencia, los procesos de evaluación son más diversos de lo deseable. En conclusión, como ocurre con muchos objetos científicos, la delimitación del complejo ira-hostilidad es un proceso lento y trabajoso y su precisión definitiva es, tal vez, una meta que no parece cercana todavía.

\section{Referencias}

Barefoot, J. C. (1992). Developments in the measurement of Hostility. En H. S. Friedman (Eds.). Hostility, coping and health. (pp. 13-21). Washington: American Psychological Association.

Boltwood, M. D., Taylor, C. B., Boutte Burke, M., Grogin, H. \& Giacomini, J. (1992). Anger report predicts coronary artery vasomotor response to mental stress in atherosclerotic segments. American Journal of Cardiology, 70, 281-285.

Booth-Kewlley, S. \& Friedman, H. S. (1987). Psychological predictors of heart disease: a quantitative review. Psychological Bulletin, 101, 343-362.

Breva, A., Palmero F. \& Fernández-Abascal, E. G. (2001). Hostilidad, apoyo y reactividad cardiaca en una situación de estrés. Ansiedad y Estrés, 7 (1), 477-502.

Buss, A. H. \& Durkee, A. (1957). An inventory for assessing different kinds of hostility. Journal of Consulting Psychology, 21, 343-349.

Buss, A. H. \& Perry, M. (1992). The agression questionnaire. Journal of Personality and Social Psychology, 63, 452-459.

Calvo, F., Alemán, S. \& Ojeda, B. (1998). Relaciones entre la hostilidad y el colesterol sérico total. Estrés y Ansiedad, 4 (2-3), 311-330.

Cookle, W. W. \& Medley, M. (1954). Proposed hostility and pharasaic-virtue scales for the MMPI. Journal of Applied Psychology, 38, 414-418.

Costa, P. T. \& Dembroski, T. (1989). Agreableness versus antagonism: explication of a potential risk factor for CHD. En Siegman \& Dembroski (Eds.). Search of coronary prone behavior: beyond type A. (pp. 4163). Hillsdale: Erlbaum.

Dembrosky, T. M., MacDougall, J. M., Costa, P. T. \& Grandits, G. A. (1989). Components of hostility as predictors of sudden death and myocardial infarction in the multiple risk factor intervention trial. Psychosomatic Medicine, 51, 514-522.

Fernández-Abascal, E. G. (1994). Intervención Comportamental en los Trastornos Cardiovasculares. Madrid: Fundación Universidad-Empresa. 
Fernández-Abascal, E. G. \& Martín, M. D. (1994). Emociones negativas II: ira, hostilidad, tristeza. En Manual de motivación y emoción. (pp. 232-247). Madrid: Centro de estudios Ramón Areces.

Fernández-Abascal, E. G. \& Martín Díaz, M. D. (1997). Sensibilidad y estabilidad temporal de índices de reactividad cardiovascular en el afrontamiento activo y pasivo. Revista Electrónica de Motivación y Emoción, 1(0). http// reme.uji.es.

Fernández-Abascal, E. G. \& Martínez-Sánchez, F. (1998). La reactividad fisiológica al estrés. Ansiedad y Estrés, 4 (2-3), 111-118.

Fernández-Abascal, E. G. \& Martín Díaz, M. D. (1999). Trastornos cardiovasculares y factores emocionales. En E. G. Fernández-Abascal \& Francesc Palmero (Orgs.). Emociones y salud. (pp. 209-232). Barcelona: Ariel Psicología.

Fernández-Abascal, E. G. \& Palmero, F. (1999). Ira y hostilidad. Aspectos básicos y de intervención. En E. G. Fernández-Abascal \& F. Palmero (Orgs.). Emociones y Salud. (p. 185-208). Barcelona: Ariel Psicología.

Fernández-Abascal, E. G., Palmero, F. \& Martín Díaz, M. D. (1998). Reactividad cardiovascular, principios y características. Ansiedad y Estrés, 4 (2-3), 195-206.

Foulds, G. A., Caine, P. M. \& Creasy, M. A. (1960). Aspects of extra e intra-punitive expression in mental illness. Journal of Mental Sciences, 106, 87-104.

Friedman, M. \& Rosenman, R. H. (1974). Type A behaviour and your heart. New York: Knopf.

Friedman, M. \& Ulmer, D. (1983). Alteration of farction patientes: sumary results of the recurrent coronary prevention project. American Heart Journal, 112, 653-665.

Garcia León, A. (1993). Patrón de conducta tipo A: descripciones e intervenciones terapéuticas. Revista de Psicología General y Aplicada, 46 (3), 307-317.

García León, A. (1999). Efectos de la hostilidad sobre la reactividad cardiovascular en paradigmas tónicos y fásicos (la Respuesta Cardíaca de Defensa). Jaén: Servicio de Publicaciones de la Universidad de Jaén.

Gordon N. F. (1998). Comprehensive cardiovascular disease risk reduction in the clinical setting. Coronary Artery Disease, 9 (11), 731-735.

Harburg, E., Erfurt, J. C., Hauenstein, L. S., Chape, C., Schull, W. J. \& Shork, M. A. (1973). Socio-ecological stress, supressed hostility, skin color and black-white male blood presure: Psichosomatic Medicine, Detroit, 41, 198-202.

Haynes, S. G. (1978). The relationship of psychosocial factors to coronary heart disease in the Framingham Study: I. Methods and risk factors. American Journal of Epidemiology, 107, 362-383.

Hoshmand, L. T. \& Austin, G. W. (1987). Validation studies of a multifactor cognitive-behavioral Anger Control Inventory. Journal of Personality Asessment, 51, 417-432.

Houston, B. K. \& Vavak, C. R. (1991). Hostility: developmental factors, psychosocial correlates, and health behaviors. Healt Psychology, 10, 9-17.

Johnson, E. H. (1990). The deadly emotions. The role of anger, hostility and aggression in health and emotional wellbeing. New York: Praeger.

Krantz, D. S. \& Durel, L. A. (1983). Psychobiological substrates of the type A behavior pattern. Health Psychology, 2, 393-411.

Lazarus, R. S. \& Folkman, S. (1984). Stress, appraisal and coping. New York: Springer.

Lazarus, R. S. (1991). From psychological stress to the emotion: a story of the changing outlooks. Anual Review of Psychology, 44, 1-21.

Leiker, M. \& Hailey, B. J. (1988). A link between hostility and disease: poor health habits? Behavioral Medicine, 14, 129-133.

Markovitz, J. H. (1998). Hostility is associated with increased platelet activation in coronary heart disease. Psychosomatic Medicine, 60, 586-591.

Martín, M. D. \& Fernández-Abascal, E. G. (1994a). Inventario de reacciones de ira de Novaco. En E. G. Fernández-Abascal. Intervención comportamental en los trastornos cardiovasculares. (pp. 76-80). Madrid: Fundación Universidad-Empresa.

Martín, M. D. \& Fernández-Abascal, E. G. (1994b). Inventario de reacciones de hostilidad de BussDurkee (BDHI). En E. G. Fernández-Abascal, Intervención comportamental en los trastornos cardiovasculares. (pp. 85-91). Madrid: Fundación Universidad-Empresa.

Martín Díaz, M. D., Jiménez Sánchez, P. \& FernándezAbascal E. G. (2000). Estudio sobre la escala de estilos y estrategias de afrontamiento (E3A). Revista Electrónica de Motivación y Emoción, 3(4). http//reme.uji.es.

Matthews, K. A. (1988). Coronary heart disease and type A behaviours: update on and alternative to the Booth-Kewley and Friedman quantitative review. Psychological Bulletin, 104, 373-380.

Matthews, K. A., Glass, D. C., Rossenman R. H. \& Bortner, R. W. (1977). Competitive drive, pattern A, and coronary heart disease: a further analysis of some 
data from the Western Collaborative Group Study. Journal of Chronic Desease, 30, 489-498.

Novaco, R. W. (1975). Anger, control: the developmente and evaluation of an experimental treatment. Lexington, MA: Lexington Books/D. C. Health.

Obrist, P. A. (1981). Cardio-vascular psychophysiology: a perspective. New York: Plenum Press.

Palmero, F. \& Codina, V. (1996). Influencia de las emociones en la reactividad cardiovascular. Valencia: Promolibro.

Palmero, F. \& Breva, A. (1998). Estrés: de la adaptación a la enfermedad. Valencia: Promolibro.

Price, V. A. (1982). Type A behavior pattern: a model for research and practice. New York: Academic Press.

Rosenman, R. H. (1974). Type A behaviour and your heart. New York: Knopf.

Rosenman, R. H. \& Palmero, F. (1998). Ira y hostilidad en la enfermedad coronaria. En Palmero, F. \& Fernández Abascal (Orgs.). (pp. 117-141). Emociones y adaptación. Barcelona: Ariel.

Rosenman, R. H., Friedman, M., Straus, R., Wurm, M., Kositchek, R., Han, W. \& Werthesen, N. T. (1964). A predictive study of coronary heart disease: the Western Collaborative Group Study. Journal of de American Medical Association, 189, 15-22.

Siegel, J. M. (1986). The multidimensional anger inventory. Journal of Personality and Social Psychology, 51, 191-200.

Smith, T. W. (1992). Hostility and health: current status of a psychosomatic hyphotesis. Health Psychology, 11, 139-150.

Smith, T. W. (1994). Concepts and methods in the studios of anger, hostility and health. En A. W. Siegman y T. W. Smith (Eds.). Anger, hostility and beart. (pp. 23-42). Hillsdale, N. J.: Lawrence Earlbaum Associates.

Smith, T. W. \& Frohm, K. D. (1985). What's so unhealthy about hostility? Construct validity and psychological correlates of the Cook and Medley Ho scale. Health Psychology, 4, 503-520.

Smith, T. W. \& Pope, M. K. (1990). Cynical hostility as a health risk: current status and future directions. Journal of Social Behavior and Personality, 5, 77-88.

Spielberger, C. D. \& London, P. (1990). Blood pressure and injustice. Psychology Today, 1-2, 49-51.

Spielberger, C. D., Johnson, E. H., Russel, S. F., Crane, R. J. \& Worden, T. J. (1985). The experience and expression of anger: construction and validation of an anger expression scale. En M. Chesney \& R. H. Rosenman (Eds.). Anger and hostility in cardiovascular and behavioral disorders. (pp. 5-30). New York: Hemisphere.

Spielberger, C. D., Krasner S. S. \& Solomon E. P. (1988). The experience, expression and control of anger. En M. P. Janisse (Eds.). Health Psychology: individual differences and stress. (pp. 89-108). New York: Springer Verlag Publishers.

Zelyn, M., Adler, G. \& Myerson, P. G. (1972). Anger self-report: an objective questionnaire for the measurement of aggression. Journal of Consulting and Clinical Psychology, 39, 340.

Enviado em março de 2003 Reformulado em maio de 2003 Aprovado em junho de 2003

Sobre os autores:

Jorge Iacovella é psicólogo e doutorando pelo programa Emociones y Salud da Universidad Nacional de Educación a Distancia (Madrid) e docente e investigador na Facultad de Psicología, Universidad Nacional de Mar del Plata.

Marisa Troglia é psicóloga e especialista en psicoterapia cognitiva e professora da disciplina Psicología Cognitiva da Facultad de Psicología da Universidad Nacional de Mar del Plata. 
